\title{
The crystal structure of the Fab fragment of a rat monoclonal antibody against the main immunogenic region of the human muscle acetylcholine receptor
}

\author{
Maria Kontou ${ }^{1,2}$, Demetres D. Leonidas ${ }^{3}$, Efstratia H. Vatzaki ${ }^{1,3}$, Panayiota Tsantili ${ }^{1}$, Avgi Mamalaki ${ }^{1}$, \\ Nikos G. Oikonomakos ${ }^{2}$, K. Ravi Acharya ${ }^{3}$ and Socrates J. Tzartos ${ }^{1}$ \\ ${ }^{1}$ Department of Biochemistry, Hellenic Pasteur Institute, Athens, Greece; ${ }^{2}$ Institute of Biological Research \& Biotechnology, \\ The National Hellenic Research Foundation, Athens, Greece; ${ }^{3}$ Department of Biology and Biochemistry, University of Bath, UK
}

\begin{abstract}
The crystal structure of the Fab fragment of a rat monoclonal antibody, number 192, with a very high affinity $\left(K_{\mathrm{d}}=0.05 \mathrm{nM}\right)$ for the main immunogenic region of the human muscle acetylcholine receptor (AChR), has been determined and refined to $2.4 \AA$ resolution by X-ray crystallographic methods. The overall structure is similar to a Fab (NC6.8) from a murine antibody, used as a search model in molecular replacement. Structural comparisons with known antibody structures showed that the conformations of the hypervariable regions H1, H2, L1, L2, L3 of Fab192 adopt the canonical structures 1, 1, 2, 1, and 1, respectively. The surface of the antigen-binding site is relatively planar, as expected for an antibody against a large protein antigen, with an accessible area of $2865 \AA^{2}$. Analysis of the electrostatic surface potential of the antigen-binding site shows that the bottom of the cleft formed in the center of the site appears to be negatively charged. The structure will be useful in the rational design of very high affinity humanized mutants of Fab192, appropriate for therapeutic approaches of the model autoimmune disease myasthenia gravis.
\end{abstract}

Keywords: antibody structure: acetylcholine receptor: X-ray crystallography.

The nicotinic acetylcholine receptor (AChR) of mammalian muscle is a ligand gated cation channel, composed of five homologous subunits in a stoichiometry $\alpha_{2} \beta \gamma \delta$ or $\alpha_{2} \beta \varepsilon \delta$ [1]. $\mathrm{AChR}$ is the target of an antibody-mediated autoimmune response, which causes the disorder myasthenia gravis (MG). Anti-AChR autoantibodies act mainly by causing AChR loss through antigenic modulation and complement activation, both mechanisms being dependent on the bivalent nature of the antibodies, resulting in failure of the neuromuscular transmission [2].

The majority of the anti-AChR antibodies in experimental rats and in sera of MG patients compete for binding to a region in the extracellular surface of the $\alpha$-subunit of the AChR, called the main immunogenic region (MIR) $[3,4]$. The approximate location of the MIR on the intact AChR has been determined, by electron microscopy, to be at the extreme synaptic end of the $\alpha$-subunit of the AChR [5]. The MIR seems to consist of a

Correspondence to S. Tzartos, Hellenic Pasteur Institute, 127 Vas. Sofias Ave., Athens, 11521 Greece. Fax and Tel.: + 3016457831 , E-mail: tzartos@mail.pasteur.gr or K. Ravi Acharya, Department of Biology and Biochemistry, University of Bath, Claverton Down, Bath BA2 7AY, UK. Fax: + 441225 826779, Tel.: + 441225826238 , E-mail: K.R.Acharya@bath.ac.uk

Abbreviations: AChR, nicotinic acetylcholine receptor; MIR, main immunogenic region; $\mathrm{MG}$, myasthenia gravis; V, variable region; $\mathrm{C}$, constant region; L, light chain; $\mathrm{H}$, heavy chain; $\mathrm{CDR}$, complementarity determining region.

Note: classification of the various domains is as follows: variable domain of light chain (VL), residues 1-107; constant domain of light chain (CL), residues 108-213; variable domain of heavy chain $(\mathrm{VH})$, residues $1-116$; constant domain of heavy chain $(\mathrm{CH})$, residues $117-214$.

(Received 9 December 1999, revised 22 February 2000, accepted 24 February 2000) group of overlapping epitopes. Although anti-MIR antibody binding is conformation dependent, some anti-MIR monoclonal antibodies (mAbs) bind weakly but detectably to denatured AChR fragments and have been used to localize a major loop of the MIR between residues $67-76$ of the $\alpha$-subunit [6,7]. The conformation of the $\alpha 67-76$ decapeptide has been studied by 2D-NMR spectroscopy [8,9]. Yet, the anti-MIR mAbs with high affinity for human AChR do not bind to denatured AChR fragments, therefore their precise epitopes are still unknown.

Anti-MIR mAbs cause AChR loss when added to cell cultures $[10,11]$ and AChR loss and symptoms of experimental MG when injected into rats [12]. Contrary to the intact antiMIR antibodies, their Fab fragments, being univalent, do not cross-link the AChR molecules, and thus they do not cause AChR loss nor MG symptoms [12]. In fact it has been shown in muscle cell cultures $[10,11]$ that these Fabs are capable of efficiently protecting the AChR against loss induced by human MG patients sera. Furthermore, it has been recently shown that such anti-MIR Fabs are also capable of protecting experimental rats against passively transferred experimental MG (D. Papanastasiou, C. Poulas and S. J. Tzartos, unpublished results), suggesting that they may be therapeutically useful.

Despite thorough investigation of the MIR, little is known about the structural characteristics of the anti-MIR antibodies. Detailed knowledge of the structure of anti-MIR Fabs will help to elucidate the molecular basis of the interaction between the AChR and anti-MIR antibodies. Furthermore this structure will be helpful in the construction of high affinity humanized mutants of the available antibody fragments for use as efficient protectors of the AChR against the pathogenic activity of anti-AChR antibodies, in trials for MG treatment.

Here, we present a structural analysis at $2.4 \AA$ resolution of the Fab fragment of the anti-MIR mAb 192. This Fab fragment has a high affinity $\left(K_{\mathrm{d}}=0.05 \mathrm{~nm}\right)$ for human AChR [13] and 
Table 1. Diffraction data statistics for Fab192 at $100 \mathrm{~K}$. Merging $R_{\mathrm{m}}$ is defined as $R_{\mathrm{m}}=\left(\Sigma_{\mathrm{i}} \cdot \Sigma_{\mathrm{h}}\left|<I_{\mathrm{h}}>-I_{\mathrm{ih}}\right|\right) / \Sigma_{\mathrm{i}} \cdot \Sigma_{\mathrm{h}} \cdot I_{\mathrm{ih}}$, where $\left\langle I>\right.$ and $I_{\mathrm{ih}}$ are the mean and $i$ th measurement of intensity for reflection $h$, respectively. $\sigma(I)$ is the standard deviation of $I$. Angle brackets denote average values.

\begin{tabular}{ll}
\hline Parameter & Value \\
\hline Space group & $\mathrm{C} 222_{1}$ \\
No. of images (degrees) & $213(159.75)$ \\
Unit cell dimensions & $a=79.7 \AA, b=110.1 \AA, c=199.5 \AA$ \\
Solvent content $(\%)$ & 43.8 \\
Resolution & $20-2.4 \AA$ \\
No. of observations & 118,352 \\
No. of unique reflections & 26,381 \\
$I / \sigma(I)$ (outermost shell) & $25.45(9,12)$ \\
Completeness (outermost shell) & $76.1 \%(51.9 \%)$ \\
$R_{\mathrm{m}}$ (outermost shell) & $0.087(0.232)$ \\
Multiplicity & 4.1 \\
Outermost shell & $2.5-2.4 \AA$ \\
Mosaicity & 0.35 \\
\hline
\end{tabular}

together with the recombinant scFv fragment, are very efficient in protecting human AChRs against binding of sera from MG patients.

\section{MATERIALS AND METHODS}

\section{Data collection and processing}

The preparation, purification and crystal growth conditions of Fab192 have been described previously [13]. The Fab fragment was purified with anion exchange chromatography and chromatofocusing and crystallized at a concentration of $12 \mathrm{mg} \cdot \mathrm{mL}^{-1}$. The crystallization conditions were $18 \% \mathrm{w} / \mathrm{v}$ poly(ethylene glycol) $6000,150 \mathrm{~mm} \mathrm{NaCl}, 100 \mathrm{~mm}$ bis-Tris/HCl $\mathrm{pH} 7.5$, $2 \mathrm{~mm}$ EDTA, at $16{ }^{\circ} \mathrm{C}$. Diffraction data to $2.4 \AA$ resolution were collected using the synchrotron radiation at Elettra Sinchrotrone (Trieste, Italy) at $100 \mathrm{~K}$. The crystals were cryoprotected by equilibration for $1 \mathrm{~min}$ in mother liquor with $25 \%$ (w/v) poly(ethylene glycol) 400. Data were integrated, scaled and merged using MARXDS and MARSCALE [14]. The crystals belong to orthorhombic space group $\mathrm{C} 222_{1}$ with unit cell dimensions $a=79.7 \AA, b=110.1 \AA, c=199.5 \AA$, with two Fab molecules per asymmetric unit and $43.8 \%$ of solvent in the crystal. Crystallographic data was collected at $100 \mathrm{~K}$ and processing statistics are shown in Table 1.

\section{Crystal structure determination}

The structure of Fab192 was determined by molecular replacement, using the murine anti-sweetener Fab (PDB entry 2cgr; anti[2N-( $p$-cyanophenyl)- $N^{\prime}$-(diphenylemethyl)-guanidineacetic acid] Fab NC6.8, IgG2b, kappa) [15], as a search model. This Fab structure was chosen on the basis of sequence similarity (62\% identity, $77 \%$ homology) and because it was solved in, relatively, high-resolution $(2.2 \AA)$.

XPLOR 3.8 [16] was used for molecular replacement searches, using data from variable resolution ranges (15.0-5.0, 10.0-4.0, and $10-3.5 \AA)$. The elbow angle of the search model was varied from the starting value in increments of $5^{\circ}$ from -17 to $20^{\circ}$ and the peaks of each search were subjected to Patterson correlation refinement, as described by Brunger [18], to obtain the final rotation angles. The entire molecule, the two domains (variable and constant) and, finally, the individual heavy and light chains in each domain, were treated as independent rigid bodies in the Patterson correlation refinement. This procedure produced an unambiguous solution, a peak $6 \sigma$ above background level, corresponding to a change in the elbow angle of $+10^{\circ}$ from the original model. A second rotation solution was not found in the cross rotation search, even when other Fab structures were used as search models, and a self-rotation search did not reveal any significant peak apart from those determined by the crystallographic symmetry. Therefore, the direct rotation function subroutine in XPLOR [19] was employed, using data from 10.0 to $3.5 \AA$. The search probe was constructed by increasing the elbow angle of the model (2cgr) by $10^{\circ}$, truncating the side chains at the $\mathrm{C} \beta$ atoms for all its residues and omitting all hypervariable regions. The peaks of this search were again subjected to Patterson correlation refinement. Under these conditions, two peaks became apparent ( $8 \sigma$ and $3 \sigma$, respectively, above background level), corresponding to the two Fab192 molecules of the asymmetric unit.

Translation searches were carried out indepedently for the two molecules, using the properly oriented and Patterson correlation refined model and data from 10.0 to $3.5 \AA$. Significant peaks emerged for both molecules $(9 \sigma$ and $5 \sigma$, respectively, above the mean) and finally, the relative $y$ translation for the second molecule with respect to the first, was found by one-dimensional traslation searches.

\section{Structure refinement}

All crystallographic refinement and electron density map calculations were carried out using the program XPLOR 3.8 [16]. After placing the two molecules in the asymmetric unit with molecular replacement, the overall orientation of the structure was subjected to a rigid body minimization in eight rigid groups, refining against data from $10 \AA$ to $3.0 \AA$ resolution. The packing of the structure was inspected visually, using the interactive computer graphics program o [20]. No steric clashes were observed, that could not be corrected by minor structural adjustments. The crystallographic $R$ factor after this stage was $0.48\left(R_{\text {free }}=0.49\right)$, at which point the Fab192 amino-acid sequence was applied to the model. Calculated phases obtained from the rigid-body refined structure were used to determine a SIGMAA-weighted [20] 2Fo-Fc electron density map. Careful examination of this map and using the amino-acid sequence of Fab192 [21] allowed the replacement of all truncated side chains in the model and the building of the misssing loops, using the program o [20]. Appropriate density was observed for most of the omitted residues.

Several alternating cycles of manual rebuilding, conventional positional refinement, and the simulated annealing method, as implemented in XPLOR [22] improved the quality of the model. Simulated annealing refinement was performed starting from $2500 \mathrm{~K}$, using ideal structure factor amplitude weights as determined by XPLOR. Individual $B$ factor refinement was carried out before computing electron density maps for interpretation. Extension of the refinement from $3.0 \AA$ (where the data are $89 \%$ complete) to $2.4 \AA$ resolution was performed in $0.1 \AA$ resolution steps, although data from 2.9 to $2.4 \AA$ were $68 \%$ complete. The inclusion of the higher resolution data in refinement allowed individual $B$ factor refinement and addition of 209 water molecules to the model. A significant improvement of the electron density maps and a drop of approximately $2 \%$ in the $R_{\text {free }}$ value indicated that inclusion of the additional data improved the model. However, the resolution of the final 

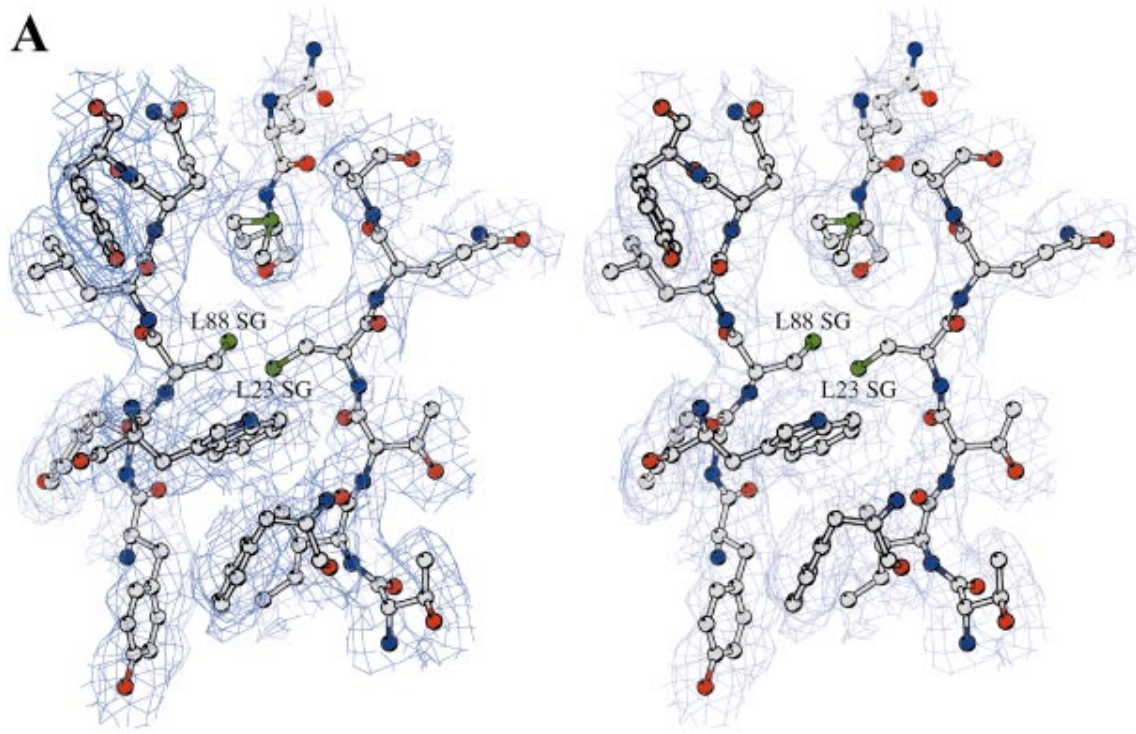

Fig. 1. Stereo diagrams of residues of the light chain of Fab192 in the vicinity of the disulphide bond between Cys23 and Cys88 (a) and residues from the CDR3 loop of the heavy chain (b) and electron densities from $2 \mathrm{Fo}-\mathrm{Fc}$ maps. The contour levels correspond to $0.13 e \cdot \AA^{-3}$ (e, electrons). The figures were produced using the program BOBSCRIPT [43].
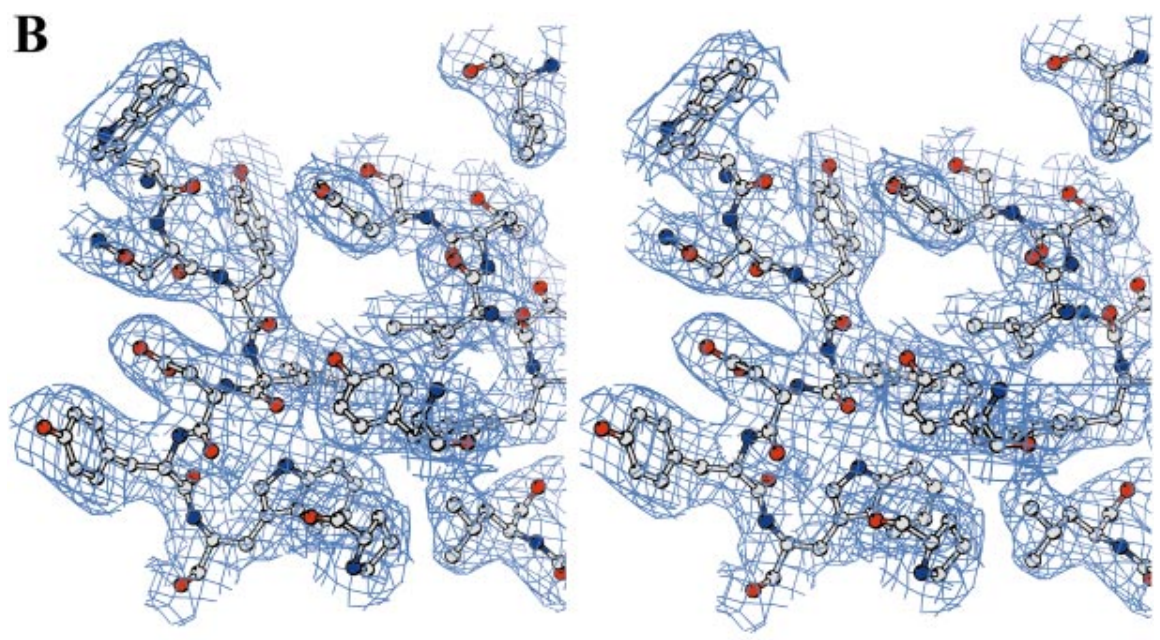

structure is nominally $2.4 \AA$ as data were $68 \%$ complete between $2.9 \AA$ and $2.4 \AA$. Bulk solvent correction, as implemented in XPLOR [23] and all observed reflections, including low-resolution data to $20 \AA$ (a total of 26381 reflections for the final cycles with no sigma cutoff) were used. The refinement process was monitored by the use of both the conventional $R$ factor and the $R_{\text {free }}$ value (based on $5 \%$ of the reflections ommited from all refinement steps) [24]. Tight noncrystallographic symmetry (NCS) restraints were used at the beginning of the refinement and they were gradually released at the final stages. Due to a small difference in the elbow angle of the two molecules of the asymmetric unit, individual NCS matrices had to be used for each of the four domains. The switch regions, connecting the variable and constant domains of either chain, were not restrained by NCS. During the final stages of refinement, water molecules were inserted into the model only if there were peaks in the Fo-Fc electron density maps with heights greater than $3 \sigma$ and they were at hydrogen bond forming distances from appropriate atoms. $2 \mathrm{Fo}-\mathrm{Fc}$ maps were also used to check the consistency in peaks. Water molecules with a temperature factor higher than $60 \AA 2$ were excluded from subsequent refinement steps. Residues where overall average $B$ factor values exceed $60 \AA^{2}$ include H101-102, H132-139, H162-165 (first molecule), and L126, L141-158,
L189-205, L208-213, H1, H131-140, H162-166, H175, H187-194, H212-214 (second molecule). Portions of the final electron density map in the region of the disulphide bond between Cys23 and Cys88 and in the region of the CDR3 loop of the heavy chain (H3) are shown in Fig. 1.

The program PROCHECK [25] was used to assess the stereochemical quality of the intermediate and the final models. The solvent accessible area of the CDRs was calculated using the program SURFACE (CCP4) [26], with a 1.7- $\AA$ probe radius and standard van der Waals radii. The structure was analyzed with the graphics program o [20]. Hydrogen bonds were assigned if the distance between the donor (D) and the acceptor (A) atoms was less than $3.3 \AA$ and if the angle D-H-A was greater than $90^{\circ}$. Van der Waals interactions were assigned for nonhydrogen atoms separated by less than $4 \AA$. The protein structures were compared using either the subroutine LSQ of the o package [20] or LSQKAB (CCP4) [26]. Surface curvature and electrostatic calculations were performed using the program GRASP [27,28].

Coordinate sets for comparison were: murine antisweetener Fab [15] (PDB code 2cgr); antibody D1.3 [29] (PDB code 1vfa). Coordinates for Fab192 (PDB code 1C5D) have been deposited with the RCSB Protein Data Bank (http:// www.rcsb.org/pdb/). 
Table 2. Refinement statistics. Crystallographic $R$ factor is defined as $R=\Sigma|| F_{\mathrm{o}}|-| F_{\mathrm{c}}|| / \Sigma\left|F_{\mathrm{o}}\right|$, where $\left|F_{o}\right|$ and $\left|F_{c}\right|$ are the observed and calculated structure factor amplitudes, respectively. $R_{\text {free }}$ is the corresponding $R$ value for a randomly chosen $5 \%$ of the reflections that were not included in the refinement.

\begin{tabular}{|c|c|c|c|c|}
\hline Parameter & \multicolumn{4}{|l|}{ Value } \\
\hline No of reflections used (free) & \multicolumn{4}{|l|}{$26381(1288)$} \\
\hline Completeness & \multicolumn{4}{|l|}{76.1} \\
\hline Residues included & \multicolumn{4}{|c|}{$1-213$ (light chain), 1-214 (heavy chain) } \\
\hline No of protein atoms & \multicolumn{4}{|l|}{6509} \\
\hline No of water molecules & \multicolumn{4}{|l|}{209} \\
\hline Final $R\left(R_{\text {free }}\right)$ & \multicolumn{4}{|l|}{$19.6 \%(30.4 \%)$} \\
\hline rmsd in bond lengths $(\AA)$ & \multicolumn{4}{|l|}{0.010} \\
\hline rmsd in bond angles $\left({ }^{\circ}\right)$ & \multicolumn{4}{|l|}{1.6} \\
\hline rmsd in dihedral angles $\left(^{\circ}\right)$ & \multicolumn{4}{|l|}{30.8} \\
\hline rmsd in improper angles $\left({ }^{\circ}\right)$ & \multicolumn{4}{|l|}{0.8} \\
\hline rms error in coordinates $(\AA)^{\mathrm{a}}$ & \multicolumn{4}{|l|}{0.32} \\
\hline \multicolumn{5}{|l|}{ Average $B\left(\AA^{2}\right)$ for residues } \\
\hline & \multicolumn{2}{|l|}{ Molecule 1} & \multicolumn{2}{|l|}{ Molecule 2} \\
\hline & light chain $(\mathrm{L})$ & heavy chain $(\mathrm{H})$ & light chain $(\mathrm{A})$ & heavy chain (B) \\
\hline Overall & 27.9 & 37.0 & 43.8 & 39.5 \\
\hline Main chain & 27.5 & 37.5 & 43.8 & 39.5 \\
\hline Side chain & 28.2 & 36.5 & 41.1 & 38.8 \\
\hline Average $B\left(\AA^{2}\right)$ for water molecules & 36.6 & & & \\
\hline
\end{tabular}

${ }^{\mathrm{a}}$ See [45].

\section{RESULTS AND DISCUSSION}

\section{Description of the structure}

The final model comprises all 213 residues of the light (L) chain and 214 residues of the heavy $(\mathrm{H})$ chain. The model also includes a total of 209 water molecules, a number consistent with the resolution and the data collection temperature $(100 \mathrm{~K})$. The model has a final conventional $R$ value of 0.196 and last recorded $R_{\text {free }}$ value of 0.304 . The $R_{\text {free }}$ value, used as a statistical cross-validation diagnostic for assessing improvement during refinement, is 0.304 , a value which is consistent with values found for other proteins at this resolution [30]. The refinement statistics of the final model of Fab192 are summarized in Table 2. The geometry of the model is good, with rms deviation from ideality of bond lengths and bond angles of $0.01 \AA$ and $1.64^{\circ}$, respectively. The Ramachandran plot [31] showed $78.7 \%$ of residues in the most favoured regions, $17.2 \%$ of residues in the additional allowed regions, $2.7 \%$ of residues in the generously allowed regions and 10 residues, which represent $1.4 \%$ of the total number, in the disallowed regions. Two of them (residue Thr51 of the CDR-L2 for both molecules of the asymmetric unit) exhibit torsion angle values that are commonly reported for residues at this position in many immunoglobulin structures [32]. The others include residues Asn30 and Glu194 of the light chain and residues Asp99 and Ser176 of the heavy chain of the second molecule are located in loops connecting $\beta$-strands which are mobile (as shown by the high $B$ factors of the corresponding regions). Ser201 and $\operatorname{Arg} 210$ of the light chain for both molecules are located in the C-terminal region of the molecule, which shows high mobility. Ser138 of the heavy chain of the second molecule is located in a region poorly supported by electron density. The equivalent region is also poorly ordered in other Fab structures [33].
Finally, Ala49 of the heavy chain of the first molecule is located on a $\beta$-strand (residues 46-52), which has a well defined electron density and is not involved in any lattice contacts, so the reasons for its unfavourable torsion angles are not clear.

The $B$ factor statistics for each chain is shown in Table 2 . In general there is good correlation between the $B$ factor and the secondary structure of the chain. The highest temperature factors in the heavy chain occur at the C-terminus and in solvent exposed loops involving residues 101-102, 132-139, and 162-165. Segment 132-139 is rarely ordered in most Fab crystal structures and has been implicated as a mediator of flexibility between segments of the antibody molecule [34]. The second molecule of the asymmetric unit has, in general, higher $B$ factors than the first, indicating that the crystal packing of this molecule is loose.

A representation of the entire structure is shown in Fig. 2. Fab192 shows the typical immunoglobulin fold. The molecule is almost completely extended and there are no contacts between the variable and constant regions of the sequence. Superposition of the structure on other Fab structures gives typical rms deviations, compared to other Fabs. The rmsd between the $\mathrm{C} \alpha$ atoms in Fab NC6.8 (the molecular replacement model) and the two molecules of Fab192 is $2.10 \AA$ and $1.99 \AA$, respectively. Based on a pair-wise comparison of the two crystallographically independent Fab molecules, only small differences can be seen. The elbow angles differ by about $2^{\circ}$ between the two molecules $\left(163^{\circ}\right.$ for molecule 1 and $165^{\circ}$ for molecule 2). The overall rmsd between the two molecules is $0.99 \AA$, while the rmsds between the light and heavy chains are $0.73 \AA$ and $0.97 \AA$, respectively. The rmsds between the variable domains are $0.52 \AA$ and $0.52 \AA$ and the rmsds between the constant domains are $0.79 \AA$ and $0.86 \AA$, respectively, for light and heavy chains. The most significant deviations occur 


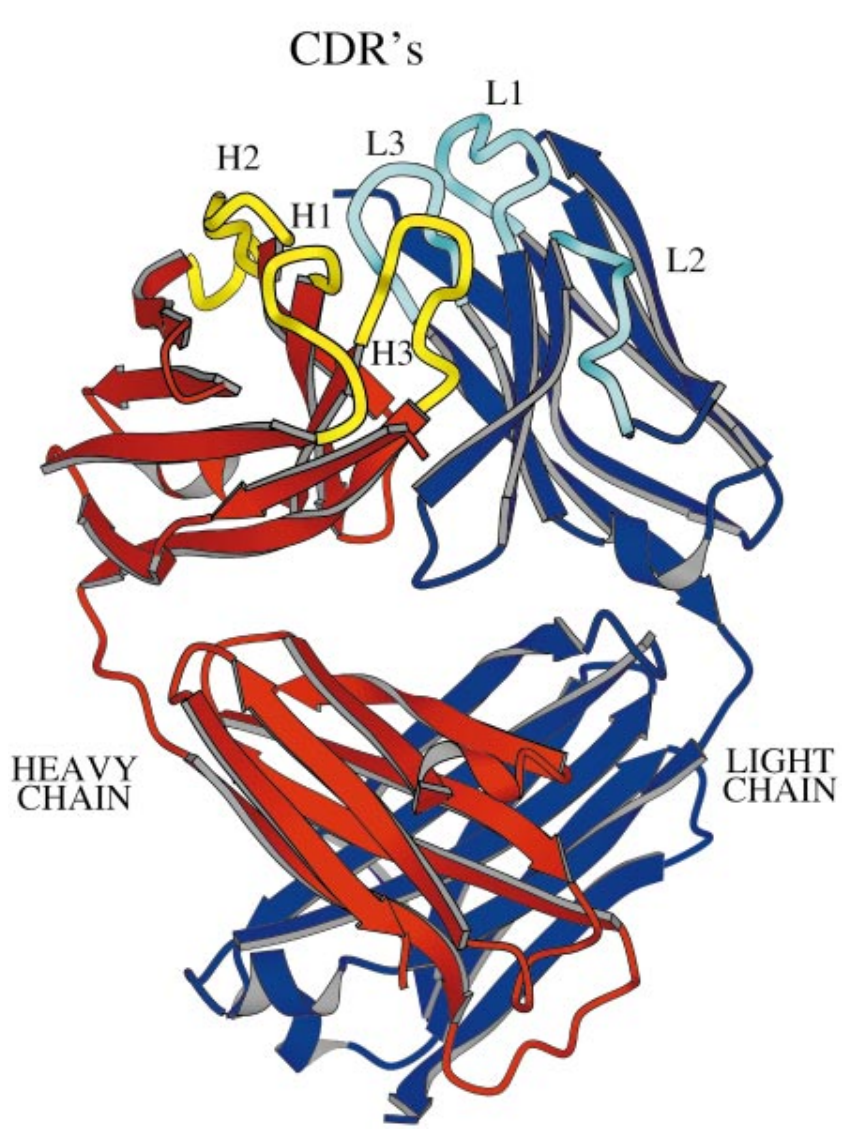

Fig. 2. A schematic diagram of the Fab192 molecule. The light chain is blue and the heavy chain is red. The positions are shown for the CDR loops. The figure was produced using MOLSCRIPT [44].

near the elbow bend $(2.1 \AA$ for residues $105-112$ of the light chain and $1.8 \AA$ for residues $109-117$ of the heavy chain) and in certain regions in the constant domains where the electron density maps are not well defined (up to $4 \AA$ for residues 130-140).

The crystal packing (Fig. 3) of the two crystallographically independent Fab molecules follows the 'head-to-tail' fashion, which has been observed for many unliganded Fabs. The dominant interactions are between the binding surface of the second molecule (mainly residues from loop regions L2 and H3) and regions of the variable domain of the first molecule (Table 3). Trp101 of H3 makes a total of 17 van der Waals interactions ( 9 nonpolar/nonpolar, 7 polar/nonpolar, and 1 nonpolar/polar). In addition, the side chain of Trp101 is involved in water mediated interactions with main and side chain atoms of Ser170 and side chain atom of Ser80.

\section{Antigen binding site}

Antigen-binding sites are formed from six loops of polypeptide, the three loops from the variable domain of the light chain (L1, L2, L3), and the three loops from the variable domain of the heavy chain $(\mathrm{H} 1, \mathrm{H} 2, \mathrm{H} 3)$. The relationships between the amino-acid sequences of the antigen-binding sites and their structures have been extensively studied [32,35-37]. It was found that, with the exception of $\mathrm{H} 3$ which is very variable in length and sequence, a canonical structure model can be established for five of the six loops. In the structure of Fab192, the quality of the electron density map in the antigen-binding region is very good and the six hypervariable loops can all be traced with high confidence (Fig. 4). A comparison of the observed conformations with those described by the canonical structure approach of Chothia et al. [36] shows that the hypervariable regions L1, L2, H1 and H2 of Fab192 are consistent with this classification. The hypervariable region L1 belongs to the canonical group 2, as indicated by the presence of the key residues L2 (Ile), L25 (Ala), L29 (Ile) and L71 (Phe), with residues 26-28 forming an extended conformation and residues 29-32 forming a distorted type II turn. The comparison of the Fab192 structure with the structure of antibody D1.3 [29], using the program LSQKAB (CCP4) [26], showed that the positions of the $\mathrm{C} \alpha$ atoms of the L1 loops deviate from their mean positions by $0.29 \AA$, indicating that the two loops are similar in their conformation. The hypervariable region L2 belongs to the canonical group 1 (key residues L48 (Ile) and L64 (Gly)), a three residue hair-pin turn (L50-L52), stabilized by two hydrogen bonds between framework residues L49 (Arg) and L53 (Ile). The superposition of the structure of Fab192 with the structure of D1.3 over residues of loops L2 gave rmsd of $0.21 \AA$ for main chain atoms. In heavy chain, the hypervariable region $\mathrm{H} 1$ follows the conformation of group 1 [key residues H26 (Gly), H27 (Phe), H29 (Leu), H34 (Val) and H97 (Arg)]. Superposition of the Fab192 and D1.3 structures over region $\mathrm{H} 1$ gave rmsd of $0.41 \AA$ for the $\mathrm{C} \alpha$ atoms. The hypervariable region $\mathrm{H} 2$ belongs to the canonical group 1 [key residue H55 (Gly)] and is three residues long (H53-H55). These three residues (Ser-Gly-Gly) form the apex of a sevenresidue turn, with the other four residues being part of the framework region. The structure is stabilized, as expected by two main-chain hydrogen bonds between H50 (Ala) and H58 (Tyr) and two main-chain hydrogen bonds between H52 (Ser), H55 (Gly) and H56 (Ser). Superposition of the Fab192 and D1.3 structures over region $\mathrm{H} 2$ gave rmsd of $0.78 \AA$ for the $\mathrm{C} \alpha$ atoms.

The hypervariable loop L3 does not follow any of the canonical forms proposed for this region, as it is only five residues long (L91-L95) and lacks the key proline residue at position L95 or L94. A Fab crystal structure, in which the L3 hypervariable loop also lacks the key proline, has been only once reported previously in the anti-CD5 Fab CRIS-1 [38]. Four out of the five residues are common between the L3 hypervariable regions of Fab192 and CRIS-1, so it is expected that the conformation of this loop will be quite similar, although the CRIS-1 atomic coordinates are not available for a comparison. The conformation found for L3 in Fab192 can still be related to the canonical form 1, as observed in D1.3 [29], despite the differences in the amino-acid composition, with rmsd values for the $\mathrm{C} \alpha$ atoms of $0.80 \AA$ (the rms deviations for canonical forms 2 and 3 are $1.54 \AA$ and $1.86 \AA$, respectively). The only L3 key residue present in Fab192 is L90 (Gln), which stabilizes the conformation with the formation, as expected, of four hydrogen bonds through its amide group: three with main-chain atoms from residues L92 (Gly), L93 (Asn), L94 (Leu) and one with $\mathrm{O}^{\gamma}$ from the sidechain of L96 (Thr).

The H3 hypervariable region of Fab192 is 10 residues long, which is considered a moderate length. Shirai et al. [39] have shown that the H3 loop conformation is influenced by the presence of a partially buried ion-pair H97 (Arg) and H105 (Asp), located at the base of a cleft in the antigen combining site. This ion pair is also present in Fab192 H3, therefore it adopts the expected bulged structure described by Morea et al. [37]. Five out of 10 residues in Fab192 H3 loop are aromatic. A 


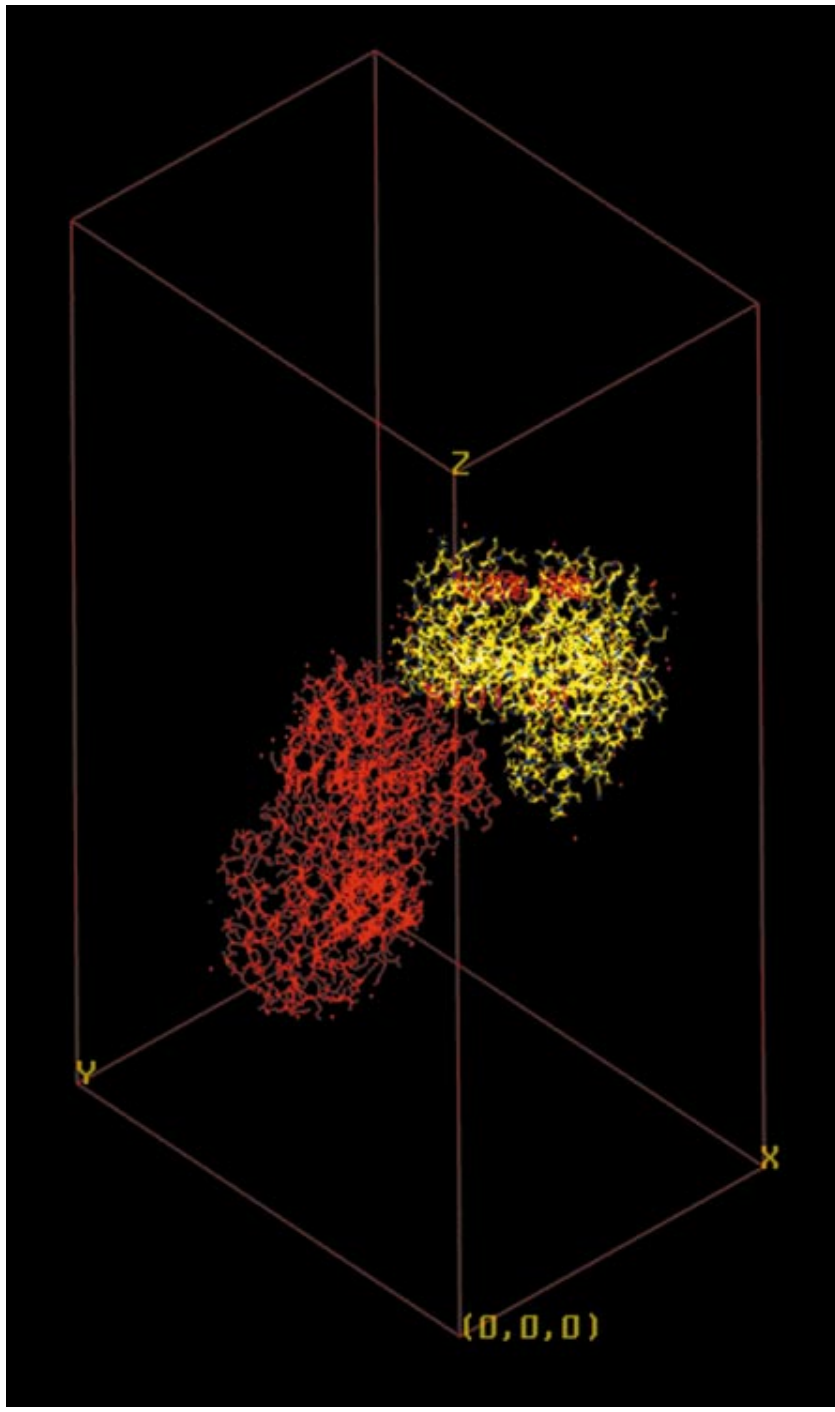

Fig. 3. A schematic diagram showing the spatial arrangement of the two molecules of Fab192 in the asymmetric unit. The first molecule is colored yellow and the second molecule red. The figure was produced using the program o [20].

study of the antigen-antibody complex structures has shown that aromatic residues play a dominant role in antigen binding [40]. Two of the aromatic residues in Fab192 hypervariable region $\mathrm{H} 3$ [H103 (Tyr) and H107 (Trp)] are important for hydrophobic packing against aromatic residues in the light chain, while the side-chains of the other three project into the cavity formed by the six CDR loops and may form part of the interaction of the antibody with the receptor.

Analysis of the correspondence between the observed canonical structure repertoire in immunoglobulin sequences and the types of antigen recognized has shown that there are two different sets of canonical structure classes: one with preference for a specific type of antigen and one with multispecific binding capabilities [41]. The combination of the canonical structures of the hypervariable loops of Fab192 for $\mathrm{H} 1, \mathrm{H} 2, \mathrm{~L} 1, \mathrm{~L} 2, \mathrm{~L} 3$ is 1121 , respectively. Vargas-Madrazo et al. [41] have found that this is a specific canonical class: $56 \%$ of antibodies belonging to it recognize protein antigens, $25 \%$ nucleic acid and $19 \%$ haptens.

The surface of the antigen-binding site (Fig. 5) is relatively planar, which is expected for an antibody against a large protein
Table 3. Intermolecular van der Waals contacts between molecule 1 and molecule 2 of Fab192. L and H, and A and B represent the light and heavy chains of molecule 1 and molecule 2 of Fab192, respectively.

\begin{tabular}{ll}
\hline Molecule 1 & Molecule 2 \\
\hline L-Leu15 CG & A-Ile53 CD1 \\
CD2 & A-Arg49 CZ,NH2,NH1, A-Ile53 CG1,CD1, \\
& B-Trp101 CZ2 \\
L-Asn77 OD1 & A-Ile53 CD1, A-Leu54 O \\
ND2 & A-Leu54 N \\
L-Ala79 CB & A-Leu56 CD1 \\
L-Glu81 CD & A-Leu56 CD2 \\
OE2 & A-Leu56 CG,CD1,CD2 \\
L-Lys106 O & B-Trp101 CZ2,CH2 \\
L-Arg107 CA & B-Trp101 CZ2,CH2 \\
CG & B-Trp101 CG,CD2,CE2,CD1,NE1 \\
CZ & B-Trp101 CB \\
NH1 & B-Trp101 CD1 \\
NH2 & B-Trp101 CB \\
L-Ala108 N & B-Trp101 CD2,CE3,CZ3 \\
CB & B-Gly100 O; B-Trp101 CE3 \\
H-Ser138 CB & B-Ser74 O \\
OG & B-Ser74 O \\
\hline
\end{tabular}

antigen [42], with an accessible area of $2865 \AA^{2}$ (calculated using the program SURFACE, CCP4 [26]). There is a cleft formed in the center of the antigen combining site which is bounded by the side chains of residues H27 (Phe), H29 (Leu), H101 (Trp) and H104 (Phe). The bottom of this cleft, formed by the side chains of residues H98 (Glu) and H99 (Asp), appears to be negatively charged. The known decapeptide of the AChR sequence that participates in the epitopes of several anti-MIR mAbs (67-76 (WNPDDYGGVK) contains two acidic and one basic residues. mAb192 does not bind to this decapeptide, nor to any other denatured AChR fragment. On the other hand, the basic K76, is followed by four more basic residues (K77, H79, $\mathrm{K} 84$, and R87) in the sequence of residues $\alpha 77-87$ (KIHIPSEKIWR). Hence, the possibility that some of these residues may

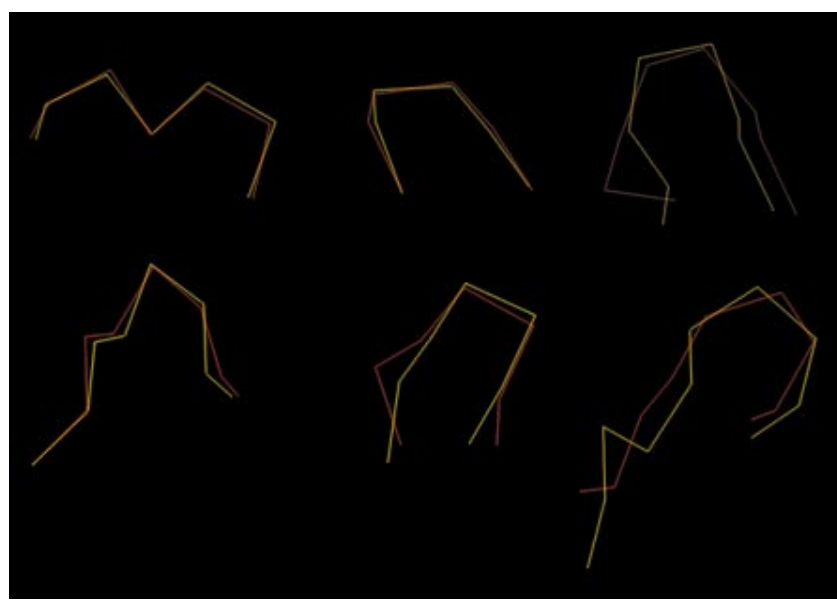

Fig. 4. Superposition of the main-chain atoms of the six hypervariable regions of Fab192 L1, L2, L3 (top) and H1, H2, and H3 (bottom), coloured yellow, to the equivalent atoms of the hypervariable regions of antibody D1.3 [29], coloured red. The figure was produced using the program o [20]. 


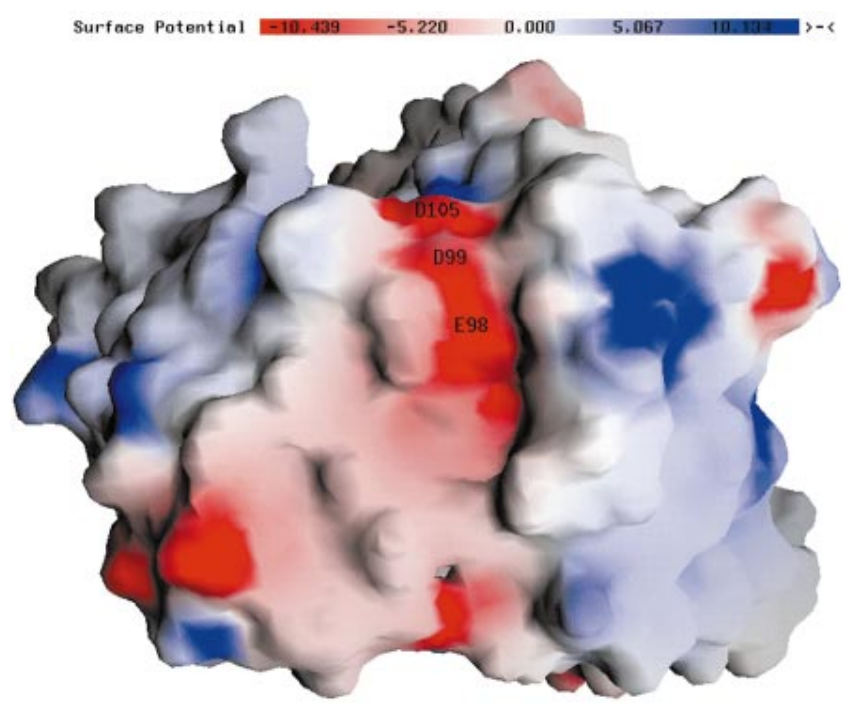

Fig. 5. View of the surface topology of antigen binding site of Fab192 molecule. The protein surface is colored according to the electrostatic potential ranging from blue $\left(\approx 10 \mathrm{kt} \cdot \mathrm{e}^{-1}\right.$, positive) to white (neutral) and red $\left(\approx-10 k t \cdot e^{-1}\right.$, negative), where $k$ is the Poisson-Boltzmann constant at temperature $t$ per electron $(e)$. The negative region created by residues from the CDR loop H3 is apparent. The figure was drawn using the program GRASP $[27,28]$.

play critical parts of the epitope for mAb192 cannot be ruled out.

In conclusion, the structure of the Fab192, the first crystal structure of an antibody against AChR, a large membrane autoantigen, provides information on structural properties required for specific recognition of the MIR of the AChR. Fab192 is very appropriate for use in therapeutic approaches of MG, after genetic manipulations. It binds at the MIR of the human AChR with an unusually high affinity [13] and very efficiently protects the AChR against binding of sera for MG patients [21]. Its recombinant $\mathrm{scFv}$ fragment has been constructed and it is similarly efficient in protecting the AChR [21]. Experiments are under way to construct the humanized mutant of this antibody fragment and increase its affinity based on the presently acquired knowledge of its antigen binding site. Although Fab192 does not bind to synthetic peptides nor to denatured AChR subunit fragments, we recently found that it binds to a refolded polypeptide corresponding to the extracellular part of the human AChR $\alpha$-subunit (T. Tsouloufis and S. J. Tzartos, unpublished results). The structural determination of the complex of Fab192 with the refolded polypeptide should further facilitate the rational design of therapeutic mutant molecules.

\section{ACK N O W LED GEMENTS}

This work was supported by grants from the Association Francaise contre les Myopathies, the contracts CHRXCT94-0547 and BIO4-CT98-0110 of the European Commission, the Greek General Secretariat of Research and Technology (EKBAN 104), grants $197 / 96$ and 214/97 of the Elettra Sinchrotrone Trieste and grant PX97-167 of the EMBL Hamburg outstation, Germany, to S. J. T. We wish to acknowledge the assistance of C. Poulas and the staff at ELETTRA, Trieste, Italy, and EMBL, Hamburg, Germany in X-ray data collection. We also thank Prof. S. Hamodrakas and Prof. E. Eliopoulos for valuable suggestions and A. Kokla for technical assistance.

\section{REFEREN CES}

1. Changeux, J.-P. (1990) Functional Architecture and Dynamics of the Nicotinic Acetylcholine Receptor - an Allosteric Ligand-Gated Ion Channel. pp. 21-168. Fidia Research Foundation Neuroscience Award Lectures, Raven Press, New York, USA.

2. Lindstrom, J., Shelton, D. \& Fugii, Y. (1988) Myasthenia Gravis. Adv. Immunol. 42, 233-284.

3. Tzartos, S.J. \& Lindstrom, J.L. (1980) Monoclonal antibodies to probe acetylcholine receptor structure: localization of the main immunogenic region and detection of similarities between subunits. Proc. Natl Acad. Sci. USA 77, 755-759.

4. Tzartos, S.J., Barkas, T., Cung, M.T., Mamalaki, A., Marraud, M., Orlewski, P., Papanastasiou, D., Sakarellos, C., Sakarellos Daitsiotis, M., Tsantili, P. \& Tsikaris, V. (1998) Anatomy of the antigenic structure of a large membrane autoantigen, the muscle-type nicotinic acetylcholine receptor. Immunol. Rev. 163, 89-120.

5. Beroukhim, R. \& Unwin, N. (1995) Three-dimensional location of the main immunogenic region of the acetylcholine receptor. Neuron $\mathbf{1 5}$, $323-331$.

6. Tzartos, S.J., Kokla, A., Walgrave, S. \& Conti-Tronconi, B. (1988) Localization of the main immunogenic region of human muscle acetylcholine receptor to residues $67-76$ of the $\alpha$-subunit. Proc. Natl Acad. Sci. USA 85, 2899-2903.

7. Papadouli, I., Sakarellos, C. \& Tzartos, S.J. (1993) High-resolution epitope mapping and fine antigenic characterization of the main immunogenic region of the acetylcholine receptor. Improving the binding activity of synthetic analogues of the region. Eur. J. Biochem. 211, 227-234.

8. Tsikaris, V., Detsikas, E., Sakarellos-Daitsiotis, M., Sakarellos, C., Vatzaki, E., Tzartos, S.J., Marraud, M. \& Cung, M.T. (1993) Conformational requirements for molecular recognition of acetylcholine receptor MIR analogues by monoclonal anti-MIR antibody: a 2D-NMR and molecular dynamics approach. Biopolymers 33, $1123-1134$.

9. Orlewski, P., Marraud, M., Cung, M.T., Tsikaris, V., SakarellosDaitsiotis, M., Sakarellos, K., Vatzaki, E. \& Tzartos, S.J. (1996) Compared structures of the free acetylcholine receptor MIR decapeptide and the antibody-bound $\left[\mathrm{A}^{76}\right] \mathrm{MIR}$ analogue. A molecular dynamics simulation from 2D-NMR data. Biopolymers (Peptide Sci.) 40, 419-432.

10. Sophianos, D. \& Tzartos, S.J. (1989) Fab fragments of monoclonal antibodies protect the human acetylcholine receptor against degradation caused by myasthenic sera. J. Autoimmun. 2, 777-789.

11. Tzartos, S.J., Sophianos, D. \& Efthimiadis, A. (1985) Role of the main immunogenic region of acetylcholine receptor in myasthenia gravis: an Fab monoclonal antibody protects against antigenic modulation by human sera. J. Immunol. 134, 2343-2349.

12. Loutrari, H., Kokla, A. \& Tzartos, S.J. (1992) Passive transfer of experimental myasthenia gravis via antigenic modulation of acetylcholine receptor. Eur. J. Immunol. 22, 2449-2452.

13. Kontou, M., Vatzaki, E.H., Kokla, A., Acharya, K.R., Oikonomakos, N.G. \& Tzartos, S.J. (1996) Characterisation, crystallisation and preliminary X-ray diffraction analysis of a Fab fragment of a rat monoclonal antibody with very high affinity for the human muscle acetylcholine receptor. FEBS Lett. 389, 195-198.

14. Kabsch, W. (1993) Automatic processing of rotation diffraction data from crystals of initially unknown symmetry and cell constants. J. Appl. Crystallogr. 26, 795-800.

15. Guddat, L.W., Shan, L., Anchin, J.M., Linthicum, D.S. \& Edmundson, A.B. (1994) Local and transmitted conformational changes on complexation of an anti- sweetener Fab. J. Mol. Biol. 236, 247-274.

16. Brunger, A.T. (1992) X-PLOR Manual - Version 3.1. A System for Crystallography and NMR. Yale University Press, New Haven, CT, USA.

18. Brunger, A.T. (1995) Patterson correlation searches and refinement. Methods Enzymol. 276, 558-580.

19. DeLano, W.L. \& Brunger, A.T. (1995) The direct rotation function: 
rotational Patterson correlation search applied to molecular replacement. Acta Crystallogr. D51, 740-748.

20. Jones, T.A., Zou, J.Y., Cowan, S.W. \& Kjeldgaard, M. (1991) Improved methods for building protein models in electron density maps and the location of errors in these models. Acta Crystallogr. A47, $110-119$.

17. Read, R.J. (1986) Improved Fourier coefficients for maps using phases from partial structures with errors. Acta Crystallogr. A42, 140-149.

21. Tsantili, P., Tzartos, S.J. \& Mamalaki, A. (1999) High affinity singlechain $\mathrm{Fv}$ antibody fragments protecting the human nicotinic acetylcholine receptor. J. Neuroimmunol. 94, 15-27.

22. Brunger, A.T., Krukowski, A. \& Erickson, J.W. (1990) Slow-cooling protocols for crystallographic refinement by simulated annealing. Acta Crystallogr. A46, 585-593.

23. Jiang, J.S. \& Brunger, A.T. (1994) Protein hydration observed by X-ray diffraction. Solvation properties of penicillopepsin and neuraminidase crystal structures. J. Mol. Biol. 243, 100-115.

24. Brunger, A.T. (1992) The free R value: a novel statistical quantity for assessing the accuracy of crystal structures. Nature 355, 472-474.

25. Laskowski, R.A., MacArthur, M.W., Moss, D.S. \& Thornton, J.M. (1993) PROCHECK: a program to check the stereochemical quality of protein structures. J. Appl. Crystallogr. 26, 283-291.

26. CCP4. Collaborative Computational Project, Number, 4. (1994) The CCP4 suite: programs for protein crystallography. Acta Crystallogr. D50, 760-763.

27. Nicholls, A. \& Honig, B. (1991) A rapid finite difference algorithm, utilising successive over relaxation to solve the Poisson-Boltzmann equation. J. Comput. Chem. 12, 435-445.

28. Nicholls, A., Sharp, K.A. \& Honig, B. (1991) Protein folding and association - insights from the interfacial and the thermodynamic properties of hydrocarbons. Proteins 11, 281-296.

29. Bhat, T.N., Bentley, G.A., Boulot, G., Green, M.I., Tello, D., Dall'Acqa, W., Souchon, H., Schwartz, F.P., Mariuzza, R.A. \& Poljak, R.J. (1994) Bound water molecules and conformational stabilization help mediate an antigen-antibody association. Proc. Natl Acad. Sci. USA 91, 1089-1093.

30. Keywegt, G.L. \& Brunger, A.T. (1996) Checking your imagination: applications of the free R value. Structure 4, 897-907.

31. Ramachandran, G.N. \& Sasisehharan, V. (1968) Conformation of polypeptides and proteins. Adv. Protein Chem. 23, 283-437.

32. Al-Lazikani, B., Lesk, A.M. \& Chothia, C. (1997) Standard conformations for the canonical structures of immunoglobulins. J. Mol. Biol. 273, 927-948.

33. Fotinou, C., Beauchamp, J., Emsley, P., de Haan, A., Schielen, W.J., Bos, E. \& Isaacs, N.W. (1998) Structure of a Fab fragment against a C-terminal peptide of hCG at $2.0 \AA$ A resolution. J. Biol. Chem. 273, 22515-22518.

34. Schneider, W.P., Wensel, T.G., Stryer, L. \& Oi, V.T. (1988) Genetically engineered immunoglobulins reveal structural features controlling segmental flexibility. Proc. Natl Acad. Sci. USA 85, 2509-2513.

35. Chothia, C. \& Lesk, A.M. (1987) Canonical structures for the hypervariable regions of immunoglobulins. J. Mol. Biol. 196, 901-917.

36. Chothia, C., Lesk, A.M., Tramontano, A., Levitt, M., Smith-Gill, S.J., Air, G., Sheriff, S., Padlan, E.A., Davies, D. \& Tulip, W.R. (1989) Conformations of immunoglobulin hypervariable regions. Nature 342, 877-883.

37. Morea, V., Tramontano, A., Rustici, M., Chothia, C. \& Lesk, A.M. (1998) Conformations of the third hypervariable region in the VH domain of immunoglobulins. J. Mol. Biol. 275, 269-294.

38. Guarne, A., Bravo, J., Calvo, J., Lozano, F., Vives, J. \& Fita, I. (1996) Conformation of the hypervariable region L3 without the key proline residue. Protein Sci. 5, 167-169.

39. Shirai, H., Kidera, A. \& Nakamura, K. (1996) Structural classification of CDR-H3 in antibodies. FEBS Lett. 399, 1-8.

40. Padlan, E.A. (1990) On the nature of antibody combining sites: unusual structural features that may confer on these sites an enhanced capacity for binding ligands. Proteins 7, 112-124.

41. Vargas-Madrazo, E., Lara-Ochoa, F. \& Almagro, J.C. (1995) Canonical structure repertoire of the antigen-binding site of immunoglobulins suggests strong geometrical restrictions associated to the mechanism of immune recognition. J. Mol. Biol. 254, 497-504.

42. MacCallum, R.M., Martin, A.C. \& Thornton, J.M. (1996) Antibodyantigen interactions: contact analysis and binding site topography. J. Mol. Biol. 262, 732-745.

44. Kraulis, P.J. (1991) MOLSCRIPT: a program to produce both detailed and schematic plots of protein structures. J. Appl. Crystallogr. 24, 946-950.

45. Luzatti, P.V. (1952) Traitement statistique des erreurs dans la determination des structures cristallines. Acta Crystallogr. 5, 802-810.

43. Esnouf, R.M. (1997) An extensively modified, Version of MolScript that includes greatly enhanced coloring capabilities. J. Mol. Graph. Model 15, 132-134. 